\title{
THE RELATIONSHIP OF THE TOXIC LYMPHOID HYPERPLASIAS TO 'LYMPHOSARCOMA AND ALLIED DISEASES *
}

\section{DOUGLAS SYMMERS, M.D.}

Professor of Pathology in the University and Bellevue Hospital Medical College, Assistant Director of Laboratories, Bellevue and Allied Hospitals NEW YORK

The dominant function of the lymphoid tissues is to arrest foreign substances brought to them in suspension and to manufacture phagocytic cells for the blood and tissue spaces. In certain situations, notably the gastro-intestinal tract, the geographical intimacy between the lymphoid tissues and an absorptive surface of enormous dimensions would seem to indicate that the lymphoid cells were placed there primarily for purposes of filtration, restraining such foreign bodies as bacteria, and filtering their toxins. That the lymphoid depots in question are a source of supply of wandering phagocytes cannot, of course, be denied, but that this function is subsidiary appears to be indicated by the fact that, in lymphatic leukemia, which is essentially a disease of the lymphoid hemopoietic system, the gastro-intestinal lymphoid follicles escape altogether, or exhibit a negligible degree of hyperplasia. The regional lymph nodes, on the contrary, such as those of the neck, axilla and groin, are actively engaged in both directions; that is to say, in the process of filtration and in the formation of lymphocytic phagocytes.

In addition to the lymphoid depots mentioned, there is an auxiliary system to be found in the form of small islands of lymphoid cells of rather wide distribution in the interstitial tissues of the thyroid, prostate, testicle, lungs, kidneys, liver and adrenal, in the subcutaneous tissues and serous membranes, and elsewhere. These deposits are sometimes so small as to escape notice in the routine histologic examination of tissues, at other times they are provided with definite germinal follicles and are easily seen. One is, nevertheless, apt to receive the impression that these lymphoid foci are functionally insignificant, and doubtless this is true. They are, however, not infrequently brought into prominence in certain diseases characterized by hyperplasia of lymphoid cells, particularly in Hodgkin's disease and lymphosarcomi, while in the liver in enteric fever they undergo increase in size in com-

* Submitted for publication Oct. 3, 1917.

* From the Pathological Laboratories of Bellevue Hospital, Director: Dr. Charles Norris. 
pany with the intestinal, mesenteric and splenic follicles, giving rise to the so-called focal necroses. In lymphatic leukemia they seldom share in the process of hyperplasia.

The spleen appears to occupy the same relationship to the arterial blood as the lymph nodes bear to the skin and mucous membranes. The presence of lymphoid follicles in the spleen and their close connection with its vascular supply, is of itself an indication that, among other things, the organ is part of the body's filtration plant. Other facts lend color to the view. Thus, the frequent occurrence of sclerotic and degenerative changes in the splenic artery suggests that this vessel is engaged in the transportation of vitiated blood to the splenic substance, while the absence of corresponding changes in the splenic vein indicates that its blood has been partially purified in the spleen before being expelled and subjected to further detoxication in the liver.

In addition, the spleen is a favorite site for separating bacteria and other parasites from the circulation, as shown by the experimental bacteremias, ${ }^{1}$ and in the same way, it is sometimes possible to demonstrate malarial parasites in scrapings or sections from the splenic pulp after repeated examinations of the peripheral blood have failed to show them. Still further confirmation is to be found in the presence of sclerotic infarctions in the spleen associated with healed or healing infective lesions of the heart valves, in which circumstances bacteria are transported during the active periods of infection from the endocardium to the splenic vessels where, acting primarily on the intima, they produce infective lesions characterized by thrombophlebitis and infarction.

Moreover, the histology of the spleen is peculiarly adapted to purposes of filtration, the distensible sinuses lending themselves admirably to the temporary pooling of blood, so that the blood itself or the endothelial cells of the sinuses, or both, may deliberately exert lytic properties on alien cells deposited in the spleen. Thus, metastasizing tumors rarely find hospitable lodgment in the spleen, even in those cases in which dissemination of tumor cells by the lymph or blood stream produces secondary growths in practically every other organ in the body. Of 298 malignant tumors studied by myself at Bellevue Hospital, ${ }^{2}$ the spleen was metastasized sixteen times, or in 5.3 per cent., and in practically every instance the secondary growths were small in both size and number. In a single exception the spleen was riddled by metastases following neoplasmic thrombosis of the splenic vein and retrograde embolism to the venous sinuses. In five cases of generalized tumor metastases the spleen was involved but once - a case of melano-

1. Bartlett and Ozarki: Jour. Med. Research, 1917, 35, 465.

2. Symmers, D.: Am. Jour. Med. Sc., 1917, 154, 2255. 
matosis in which there were three secondary deposits - and yet, in all of these cases, the spleen must have been visited on innumerable occasions by tumor cells whose vegetative properties were no less exalted than in those which were delivered to and successfully inoculated in other tissues.

Finally, it may be recalled that, in disturbances of the bone marrow, such as occur in myelogenous leukemia and Hodgkin's disease, fully formed myeloplaxes are not uncommonly discharged into the circulation and separated out in the splenic sinuses, a finding which has also been observed in experimental infection of certain lower animals by the bacillus of hog cholera, pyocyaneus and anthrax, and after repeated artificial hemorrhages. ${ }^{3}$

\section{THE REACTION OF THE LYMPH NODES TO IRRITATION}

The lymph nodes are called on to deal with at least two varieties of extraneous material, namely, solid particles, such as coal dust and other pigments, the bodies of bacteria, cell débris and the like, and soluble irritants in the form of toxins derived from the skin and mucous membranes or formed in the lymph nodes by living microorganisms. The cellular reactions in the lymph nodes vary according to the nature of the foreign bodies offered to them for disposal. In the case of insoluble particles, such as coal dust, the reaction consists in fibrotic changes in the interstitial framework, usually with, but sometimes without, corresponding numerical increase in the endothelial cells of the sinuses. The lymphoid cells maintain a passive attitude, but, as time goes on and the sinuses become occluded by desquamated endothelium and pigment granules, or are encroached on by the growing connective tissues, the flow of lymph is impeded and the lymphoid cells suffer atrophy and diminution in numbers.

In the case of bacteria the reaction in the lymph nodes differs with the nature of the invading parasite. For example, the typhoid bacillus, which combines with the bacillus of tuberculosis and the spirochete of syphilis to form a group of micro-organisms expressing an inimical predilection for lymphoid tissues, acts on the lymph nodes in such fashion as to produce necroses, together with hyperplasia of endothelium and of lymphoid cells. In the case of the tubercle bacillus, the most frequent change consists in the formation of tubercles, usually of the so-called epithelioid type, a reaction which is a deliberate attempt to limit the activity of a foreign body by erecting a structural barrier between it and healthy tissues. At the same time the lymphoid tissues in the immediate vicinity of the tubercle, or even throughout the node,

3. Opie: Am. Jour. Med. Sc., 1904, 126, 988; Pugliese: Fortschr. d. Med., $1897,15,727$. 
undergo hyperplasia, representing a response to the action of toxins derived from the bacillus - a type of biologic phenomenon the significance of which was first clearly recognized by Weigert ${ }^{4}$ who, on the basis of observations of this sort, postulated the doctrine of regenerative over-production of cells. He advanced the view that physiologic structure and function depend on equilibrium maintained by the mutual restraint of cells, and that destruction of a cell or group of cells releases restraint to an extent sufficient to disturb the equilibrium of the remaining cells, in this way permitting them to exhibit abnormal proliferative energies and to produce new cells in abundant excess of those actually required to compensate for loss. Hyperplasia, therefore, is not a direct, but an indirect result of irritation, the irritant serving to destroy cells and not to stimulate those that remain in the direction of regeneration. The doctrine of regenerative overproduction finds a corollary in the process of paralytic and degenerative hypersecretion, which is of frequent occurrence in pathologic conditions. For example, section of the corda tympani nerve results in focal degeneration and necrosis of the nuclei of the submaxillary gland, and this, in turn, is followed by abundant hypersecretion of saliva. The same process is illustrated by various inflammatory reactions, notably the catarrhal lesions, which are attended by extensive degeneration of cells and by hypersecretion of mucus. Even in physiologic conditions the process of degenerative hypersecretion is sometimes to be observed, the overproduction of milk by the lactating breast being accompanied by destructive changes in the cells of the galactiferous tubules. It is apparent, therefore, that, in reacting to injury as well as in responding to physiologic demands, nature is apt to be prodigal not only of cells but of secretions as well.

Weigert's doctrine of overproduction is abundantly illustrated; in fact, examples are far too numerous to permit of more than passing mention at this time. Suffice it to recall the excessive regeneration of tubules in the lactating breast and in chronic interstitial mastitis, as well as in certain forms of adenomatoid hyperplasia of the thyroid, stomach, colon, prostate, liver and other organs. In all of these conditions not only is the supply of new cells greatly in excess of the number lost, but the new cells constitute a menace, since they approach a type of architecture in which equilibrium between structure and function is unstable, and, unless every source of irritation is removed, growth is apt to proceed beyond the control of the body and a malignant tumor result. The doctrine is likewise applicable to the lymphoid tissues, among which excessive regeneration of cells occurs in a variety of circumstances attended by the presence in the tissues

4. Weigert: Verhandl. d. Gesellsch. d. Deutsch. Naturforsch. u. Aerzte. Part 1, September, 1896, 121. 
of toxic substances which act as irritants. Thus, the injection of tetanus toxin into the sciatic nerve is followed by hyperplasia of the lymph nodes in the vicinity of the nerve trunk, and, in certain nonulcerative malignant growths, hyperplasia of the regional nodes is not infrequently demonstrable microscopically, due, undoubtedly, to the absorption of irritant products. In this connection, the experiments of Flexner ${ }^{5}$ are of great significance. He has shown, for example, that the injection of lymphotoxins in geese and guinea-pigs, when carried out over a long period, is followed by extraordinary hyperplasia of the lymph nodes throughout the body.

Tuberculosis, however, furnishes the best examples of toxic lymphoid hyperplasia. There is a form of tuberculous lymphadenopathy consisting of diffuse hyperplasia of lymphoid cells without any suggestion of tubercles, the establishment of the nature of the process depending on the detection of tubercle bacilli in the enlarged nodes, on the experimental reproduction of tuberculosis in susceptible animals following the injection of emulsified tisstues, or on comparison with preceding or subsequent lesions in the same type of tissue in the same individual. A case of this description occurred in a 6-year-old boy at the New York Hospital. The patient presented an enormons mass on one side of the neck. Removal and microscopic examination of the enlarged nodes showed tubercles in practically all of them. Several months later the nodes on the opposite side became enlarged following an attack of diphtheria. In these the only detectable change consisted in extensive and diffuse hyperplasia of lymphoid cells. Neither tubercles nor tubercle bacilli were found in spite of an exhaustive attempt to learn the nature of the lesion, and whether it was a form of tuberculosis, or whether it was due to the absorption of toxic products from the diphtheritic membrane, was never satisfactorily determined.

It is interesting to observe that tuberculosis is held to be responsible for a certain percentage of all cases of hyperplastic tonsillitis, a view which has been challenged by certain observers on the basis of failure to find tubercles in the enlarged tonsils. In guinea-pigs, however, it has been found that injection into the tonsil of given strains of tubercle bacilli results in marked lymphoid hyperplasia, not alone in the tonsils, but in the regional nodes, and this without the formation of tubercles in either locality, tubercle bacilli, however, being demonstrable, by appropriate methods of staining, weeks or even months later. It follows, I think, that certain cases of apparently simple hyperplastic tonsillitis in human beings might be ascribed to the same cause.

5. Flexner: Univ. Penn. Med. Bull., 1902, 15, 287. 
Finally, there is a variety of disseminated lymphoid hyperplasia ${ }^{6}$ of tuberculous origin characterized by involvement of the lymph nodes throughout the body and by participation of the lymphoid structures of the stomach and spleen without the occurrence of tubercles in any of the localities named.

In connection with the same general subject I may cite the case of a woman, 55 years of age, who, about a year prior to this report presented a mass on the right side of the neck that was about the size of an adult fist. The growth projected into the side of the mouth, displacing the tonsil backward. Both the skin and mucous membrane covering the mass were intact. A portion of the growth was removed and sent to me for microscopic examination. I returned a diagnosis of lymphosarcoma, based partly on the microscopic appearances and partly on the fact that Gram-Weigert and Levaditi preparations and sections stained by Hermann's method for tubercle bacilli were negative. The patient was regarded by the surgeon as inoperable, and she was subjected to treatment by radium. The growth disappeared completely. In the light of this astonishing result I submitted the original microscopic preparations to two disinterested pathologists, both of whom concurred in the diagnosis of lymphosarcoma. Whether the diagnosis was correct or not, however, is largely a matter of scientific interest, and does not modify the fact that the mass was a localized lymphoid hyperplasia, apparently of toxic origin, the cells growing with such rapidity as to jeopardize life.

\section{LYMPHOSARCOMA}

In the necropsy service at Bellevue Hospital in the past ten years there have been twelve cases of lymphosarcoma, which, taking into account the fact that our necropsy records exceed 5,500, indicates that the lesion is not a common one. Eleven of the twelve cases occurred in males. In six of the cases the growth was first observed in the cervical lymph nodes; in three the changes observed postmortem suggested that the process commenced in the lymphoid remains of the thymus gland, and the remaining three in the small intestine. Some of the cases exhibited features which are considered worthy of presentation at length.

Lymphosarcoma is essentially a growth of regional distribution, although occasionally it displays an extraordinary faculty for bringing about diffuse lymphocytic infiltration of remote viscera. At other times it is associated with variable numbers of lymphoid nodules in such organs as the kidney, liver and suprarenal capsule where, in normal conditions, lymphoid cells are present only in the form

6. Coley and Ewing: Proc. New York Path. Soc., New Series, 1909, 10, 145. 
of foci so minute as scarcely to attract notice on microscopic examination. To the unaided eye these nodular "metastases" in lymphosarcoma appear to be well circumscribed, but, on microscopic examination they fade into the surrounding tissue spaces in such gradual fashion as to indicate hyperplasia of pre-existing lymphomas rather than foci springing from transplanted cells derived from a remote growth.

Anatomically, the lymphosa rcomas are divisible into five groups, one (a) characterized by diffuse infiltration of tissues, more especially of paired organs; a second, $(b)$ involving regional collections of superficial lymph nodes; a third, $(c)$ implicating the lymphoid structures of the thorax, notably the remnants of the thymus gland and the lymph nodes at the root of the lung; a fourth, $(d)$ the lymphoid tissues of the abdomen, including the stomach, intestine, spleen and lymph nodes, and, fifth, $(e)$ there is a variety of leukosarcoma, described by Sternberg, which is characterized by lymphoid tumors in unusual situations, the growth pouring its lymphocytes into the blood in such quantities as to constitute a leukemia. In this category belongs, also, I believe, the disease commonly known as chronic lymphatic leukemia.

\section{SYMMETRICAL LYMPHOSARCOMA}

(a). Mikulicz' Disease.-The implication of paired organs by lymphosarcoma is well shown by a lesion first fully described by Mikulicz. It usually commences as an infiltrative overgrowth of the lymphoid cells normally existing in the stroma of the lacrimal glands, and is manifested by symmetrical enlargement of the outer two-thirds of the upper eyelids, followed by symmetrical enlargement of the parotid and submaxillary glands. In the case originally described by Mikulicz the histologic changes were characteristic of lymphosarcoma, small collections of closely packed lymphoid cells being supported by delicate trabeculae of connective tissue. Precisely the same combination of symmetrical lacrimal, parotid and submaxillary adenopathy has since been observed in lymphatic leukemia, the change depending on diffuse infiltration of lymphocytes.

(b). Symmetrical Conjunctival Lymphosarcoma. - A condition related to Mikulicz' disease is to be found in bilateral lymphosarcoma of the conjunctival lymphoid follicles - a lesion which does not appear to have been described previously. The case which came under my observation was that of a man, 37 years of age. The palpebral conjunctiva of the right eye was greatly swollen and thrown into folds which caused eversion and depression of the lower lid and encroached on the ocular conjunctiva to the lower level of the cornea. The conjunctiva of the opposite eye was similarly affected, but to a less extent. 
Microscopic examination revealed the histologic picture of lymphosarcoma with neoplasmic infiltration of the tissues of the lid.

(c). Symmetrical Lymphosarcoma of the Paired Viscera. - The tendency of lymphosarcoma to bring about symmetrical neoplasmic infiltration of certain tissues is shown by bilateral invasion of the mammae, ovaries, testicles, suprarenal capsules and certain paired members of the bony system - a remarkable capacity which it shares with the so-called chronic lymphatic leukemia and its alleged companion lesion, pseudoleukemia. ${ }^{7}$ One of the most striking of all the symmetrical infitrations in lymphosarcoma, however, is to be seen in the kidneys, in which vast hordes of tumor cells penetrate the connective tissue framework and produce enormous enlargement of the organs without correspondingly extensive or severe microscopic alterations in the tubular epithelium or very profound disturbances in the excretory function of the kidney. Here, again, lymphosarcoma is not alone in producing changes of the sort described, since an identical lesion exists in the kidney in the so-called pseudoleukemia, having, in fact, been observer by Cohnheim in his original case. ${ }^{8}$

Three examples of symmetrical lymphosarcomatous infiltration of the kidneys occurred in the Bellevue Hospital necropsies, in two of which the original focus of growth was apparently in the lymphoid remains of the thymus gland, while the third was associated with lymphosarcoma of the small intestine. In the Bellevue cases the several clinical histories and necropsy protocols have been abbreviated as much as is consistent with an intelligible presentation, and are as follows:

CASE 1.-The patient was a man, aged 26 , a butcher by trade, who was admitted to the hospital complaining of shortness of breath. He said that three weeks before admission he suddenly became short of breath and subject to a severe cough attended by pain in the chest and expectoration. On admission it was noted, in addition, that the fingers were cyanotic, that the subcutaneous tissues were edematous and that ascites was marked. Repeated examinations of the sputum for tubercle bacilli were negative. The temperature was normal throughout. The urine showed a trace of albumin and hyaline and granular casts. The blood contained 13,000 leukocytes, of which 34 per cent. were lymphocytes. Physical examination revealed slight bulging and dulness over the lower right side of the chest and dulness beneath the right infraclavicular region and between the scapulae. The area of cardiac dulness was increased, the apex beat was neither visible nor palpable and the heart sounds were muffled.

Necropsy (1483). - The body was that of a man, 26 years of age. On section the subcutaneous tissues were universally edematous. The peritoneum was thickened and opaque and enclosed several liters of turbid, yellowish fluid. On removing the sternum the anterior mediastinum was found to be occupied by a growth which, in a general way, conformed to the shape of the thymus gland, and measured 9 by 13 by $10 \mathrm{~cm}$. The mass extended downward as far as the level of the auriculoventricular groove and upward to within a short

7. Fabian: Beitr. z. path. Anat. u. allg. Path., 1912, 53, 491.

8. Cohnheim: Virchows Arch. f. path. Anat., 1865, 33, 451. 
distance of the lower border of the thyroid gland. Laterally it infringed on the root of the left lung, bands of tumor tissue infiltrating the connective tissue planes along the larger bronchi for a distance of several centimeters. On section, the growth was made up of yellowish white tissue which was divided into lobules of variable size by bands of dense, whitish connective tissue. The substernal, peribronchial, peritracheal and lower cervical lymphnodes were enlarged, discrete, the largest approximating the size of a cherry, and, on section, the cut surface was bulging.

Heart: The pericardium contained about 200 c.c. of turbid, yellowish fluid with fibrin flocculi, and both layers were covered by fibrinous exudate. The heart was greatly increased in size and the epicardial fat was diffusely infiltrated by grayish yellow tissue similar to that of the growth in the region of the thymus gland. The walls of both ventricles were enormously thickened, rigid, and largely replaced by grayish yellow tumor tissue, islands of reddish musculature being preserved at scattered intervals. The pleura covering the parietal and diaphragmatic surfaces of the right lung measured $0.5 \mathrm{~cm}$. in thickness and was adherent to the chest wall, pericardium, and diaphragm through the medium of infiltrated tumor tissue.

Kidneys: The kidneys were massively enlarged and weighed together 1,300 gm. Each measured 17 by 8.5 by $8 \mathrm{~cm}$. The cortices were unusually broad and grayish yellow in color, standing out in marked contrast to the dark red pyramids. On section the cut surface was smooth, pale, the substance bulging and glazed. The mesenteric and retroperitoneal nodes were enlarged to the extent of from 1 to $4 \mathrm{~cm}$.

Anatonic Diagnosis.-Lymphosarcoma of the anterior mediastinum corresponding to the lymphoid remains of the thymus gland; lymphosarcomatous infiltration of the peribronchial connective tissues of the left lung and of the parietal pleura of the right lung, of the pericardium and both ventricles of the heart; serofibrinous pericarditis; massive symmetrical lymphosarcomatous infiltration of the kidneys; lymphosarcomatous hyperplasia of the substernal, peritracheal, peribronchial, lower cervical, mesenteric and retroperitoneal lymph nodes.

Microscopic Findings.-Microscopic examination of the growth in the region of the thymus showed the presence of innumerable large and small islands of lymphocytes separated by irregularly distributed bands of fibrous tissue. The lymphocytes in the islands showed no arrangement comparable to the delicate alveolar network so frequently seen in lymphosarcoma, but were closely packed in sheetlike arrangement. The connective tissue planes of the heart were richly infiltrated by tumor cells, the intervening muscle fibers being atrophied or completely destroyed and replaced by broad sweeps of lymphocytic cells. The connective tissues of the cortex of the kidneys were invaded to an extraordinary degree by lymphocytes, the tubules being widely separated and the glomeruli surrounded. The tubular epithelium was granular and swollen. The medulla was free. The architecture of the lymph nodes was completely lost and replaced by dense, diffusely arranged lymphocytes which infiltrated the capsule and could be seen streaming off into the surrounding tissue spaces. The other viscera revealed no microscopic changes of note.

CAse 2.-The patient was a boy, aged 17, who, on admission to the hospital, said that two weeks previously he suddenly became short of breath and was seized by dull, constant pain in the upper part of the chest, attended by cough and expectoration. The urine contained a trace of albumin, but no casts. Frequent examinations of the sputum for tubercle bacilli were negative. The sputum was blood tinged. Both legs were edematous and the face and neck were cyanotic. Physical examination of the chest showed marked flatness extending from the clavicles downward, and thoracentesis was attended by withdrawal of enormous amounts of blood tinged fluid. At this time the heart sounds were clear. The abdomen was distended by fluid. 
Necropsy (2836). - The body was that of a boy, 17 years of age. The abdomen was prominent and, on section, released a large amount of faintly blood tinged fluid. Both lower extremitics were edematous. On opening the thorax both pleural cavities were found to contain large quantities of slightly blood tinged fluid, compressing the lungs, which were displaced upward. Lying in the anterior mediastinum corresponding to the position of the thymus gland was a large, pale or yellowish, firm mass which extended upward as far as the lower level of the neck and downward in front of the pericardium to the level of the auriculoventricular groove.

Heart: The pericardium was distended by a large amount of bloody fluid. The parietal pericardium was thickened, and strewn over the visceral layer was a collection of reddish, shaggy, fibrinous exudate. The heart muscle in the upper part of the left ventricle was fleshy in appearance.

Kidneys: Both kidneys were increased in size, measuring 15 by 10 by 8 $\mathrm{cm}$., weighing together $1,040 \mathrm{gm}$. The form was well preserved and the surface was extremely pale, the cortex broad, fleshy, and the markings obscured.

The retroperitoneal and lower cervical lymph nodes were slightly enlarged. The bone marrow presented numbers of scattered, whitish areas.

Anatomic Diagnosis.-Lymphosarcoma of the anterior mediastinum, corresponding to the region of the thymus gland; lymphosarcomatous infiltration of both kidneys; lymphosarcomatous hyperplasia of the cervical and retroperitoneal lymph nodes and of the pericardium and the superficial muscular planes of the left ventricle of the heart; hemorrhagic serofibrinous pericarditis; ascites; bilateral hydrothorax.

Microscopic Examination.--The histology of the growth found in the anterior mediastinum in the position of the thymus gland showed a scanty connective tissue framework of irregular distribution, in the meshes of which were enormous numbers of closely packed lymphoid cells. The architecture of the lymph nodes was entirely obscured by diffuse overgrowth of identical cells. The epicardium was covered by a thick layer made up of a superficial stratum of fibrin, below which was a quantity of richly vascularized granulation tissue densely sown with lymphoid cells. Below this, in turn, was a zone composed exclusively of lymphoid cells, running downward from which into the intramuscular planes of the heart was a rich infiltrate, which ceased, however, a few millimeters beneath the surface. The bone marrow was richly strewn with lymphocytes.

From the pathologic standpoint the two cases are interesting as emphasizing the occurrence of massive lymphosarcomas on the basis of the lymphoid remnants of the thymus gland, the growths giving rise, clinically, to detectable signs of intrathoracic pressure, including changes in the pericardium due to neoplasmic infiltration and embarrassment of the heart's action from a like cause. The massive symmetrical infiltration of the kidneys is likewise interesting, particularly in view of the few changes referable to the secretion of urine.

CASE 3.-In a third case encountered at Bellevue Hospital the patient, a man, aged 56, was admitted with the classical signs of cardiac decompensation, death being due to croupous pneumonia. The urine contained a trace of albumin and a few hyaline and granular casts. Beyond this the clinical history contained nothing of interest in the present connection. At necropsy the middle of the jejunum presented two infiltrated, whitish elevations, $4 \mathrm{~cm}$. in diameter that extended around the intestine in bracelet fashion. They were separated from one another by several centimeters of healthy mucosa. A similar circumferential infiltrated band, $1 \mathrm{~cm}$. in diameter, was found in the 
middle of the ileum, the center of the band, corresponding to the mesenteric attachment, being ulcerated.

Kidneys: The kidneys were enlarged and weighed together $630 \mathrm{gm}$. They were yellowish white and, on section, the cortices were broadened and the markings were obliterated by cream colored tissue. Numerous minute whitish specks were visible in the cortex.

Anatomic Diagnosis.-Lymphosarcoma of the small intestine; symmetrical infiltration of the adrenals and kidneys; lymphosarcomatous foci in the liver.

Microscopic Examination.-The histology of the growth in the intestine showed scattered areas of superficial ulceration and great thickening of the wall due to infiltrating lymphocytes. The kidneys displayed enormous numbers of lymphocytes invading the intertubular spaces from cortex to pelvis. The malpighian follicles and tubular epithelium appeared to be well preserved. In the liver were innumerable minute lymphocytic foci, most of them lying in the connective tissue at the periphery of the lobules. The adrenals revealed large numbers of lymphocytes arranged in islands or scattered diffusely through both cortex and medulla.

In all of the three cases just synopsized the kidneys exhibited remarkable increase in size due to infiltration of hordes of tumor cells in the connective tissue framework, with or without attendant granular changes in the epithelium of the convoluted tubules, while the urinary changes were insignificant. It is not at all clear why a lymphosarcoma in such a remote locality as the upper thorax, or even in the intestine, should bring about diffuse symmetrical invasion of one or more sets of organs in distant parts. The only possible explanation seems to me to be that, in these circumstances, tumor cells are displaced at intervals from the parent growth and filtered out as a result of the presence in the recipient tissues of a chemotactic force, since, in the very nature of things, it is hardly conceivable that the resident lymphomas could occasion such widespread hyperplasia. In expressing this predilection for the paired viscera the lymphosarcoma is not alone. The so-called Krukenberg tumor, which is a primary carcinomatous growth springing from the parietal cells of the fundus glands in the stomach, commonly causes diffuse symmetrical invasion of the ovaries, and the same selective action is true of certain tumors which metastasize the suprarenal capsules, while the kidneys themselves are occasionally the seat of symmetrical metastases from tumors of the connective tissue series other than the lymphosarcomas.

\section{PSEUDOLEUKEMIA AND ALLIED CONDITIONS}

Shortly after Virchow described lymphatic leukemia, Cohnheim ${ }^{8}$ recorded a case which presented identical anatomic characteristics without changes in the blood, and for this disease he suggested the name of pseudoleukemia. The designation has since been misapplied in various quarters and employed synonymously with Hodgkin's disease, from which, however, pseudoleukemia is readily distinguished on microscopic 
exanination. In Cohnheim's case the patient was a man, aged 24, who suffered from anorexia, vomiting and repeated attacks of epistaxis. The leukocytes were diminished in number. At necropsy, in addition to enlargement of the cervical, inguinal and retroperitoneal lymph nodes, the spleen was found to be greatly enlarged, measuring 25 by 17 by $8 \mathrm{~cm}$., and the pulp was richly infiltrated by lymphoid cells. The kidneys were also increased in size, measuring 17 by 6 by $5 \mathrm{~cm}$., and were infiltrated by lymphoid cells from cortex to pelvis.

In addition to the variety of pseudoleukemia described by Cohnheim, a related lesion of the gastro-intestinal lymphoid structures has been recognized. As far as I have been able to learn, the gastrointestinal form is rare, only twelve cases having been found in the literature to date. ${ }^{9}$ The lesion is characterized by extraordinary hyperplasia of the gastro-intestinal lymphoid structures, myriads of enlarged follicles stretching from the cardiac end of the stomach to the anal margins, causing great thickening and rigidity throughout. The ind;vidual follicles vary in size, and are so closely packed as scarcely to exhibit a hair's breadth between them. The thoracic and superficial lympin nodes are usually involved, but not nearly in the same relative proportions as the follicles in the stomach and intestine. The abdominal nodes, however, are apt to show marked changes. The spleen is almost invariably enlarged, often approximating the splenomegaly of lymphatic leukemia. The lymphoid cells in the blood are normal or approximately so- a fact which permits instant differentiation between the true and the so-called pseudoleukemia. Anatomically, the gastrointestinal lesion in question belies the title of pseudoleukemia to the extent that, in true lymphatic leukemia, the lymphoid structures of the gastro-intestinal tract rarely, if indeed they ever, undergo a degree of hyperplasia even remotely comparable to that just described. In fact, in nineteen cases of chronic lymphatic leukemia of which $I$ have records, the gastro-intestinal lymphoid structures were practically unchanged.

In view of the extremely vague relationship of pseudoleukemia to lymphatic leukemia, and because of the anatomic distribution of the changes in the lymphoid apparatus in the so-called pseudoleukemia, and because of the fact that identical or related changes are encountered in the same situations in lymphosarcoma, I am of the opinion that pseudoleukemia should be discarded as a misleading and inappropriate designation, and that the lesion so named should be included among the lymphosarcomas.

9. Symmers, D.: The ARchives Int. Med., 1909, 4, 218; Shoemaker: New York Med. Jour., Jan. 1, 1910. 
Chronic Lymphatic Leukemia. - Chronic lymphatic leukemia is a disease of the lymphoid system characterized by widespread and practically simultaneous enlargement of certain groups of lymph nodes, notably those of the cervical and axillary regions, the peribronchial nodes, and the spleen. It is attended by enormous preponderance of lymphocytes in the blood, the cells being poured into the circulation from the hyperplastic lymph nodes, by the presence of huge numbers of infiltrating lymphocytes in the blood vessels and lymph spaces throughout the body, and by enlargement of the splenic follicles and diffuse infiltration of the pulp, the sinuses sometimes being so crowded with lymphocytes as to constitute lymphoid thrombi. How much of the latter is due to the formation of lymphoid cells in the splenic follicles and how much to deposition from the blood, is impossible to say. The liver is almost always greatly increased in size, its sinusoids are strewn with lymphocytes and circumscribed lymphocytic foci are present at frequent intervals. Finally, the bone marrow, which, in normal circumstances, is practically devoid of lymphocytic foci, is almost completely replaced, myriads of lymphocytes occupying the marrow spaces, choking and distending the capillaries. In a word, lymphatic leukemia is primarily a disease of the lymphoid system, and the blood stream, viscera, and bone marrow are secondarily infiltrated by cells identical with those encountered in the lymphosarcomas - all of which is in keeping with the conception of a primary neoplasm of the lymph nodes. Reasoning by analogy with generalized toxic lymphoid hyperplasia in tuberculosis, it appears to me that chronic lymphatic leukemia may well represent an autonomous process due to a self-perpetuating lymphotoxin origin, that it belongs to the category of lymphosarcomas, and is an example of the applicability of Weigert's doctrine of regenerative overproduction. The interpretation of lymphatic leukemia as a neoplasm attended by circulating metastases is substantiated by Sternberg's ${ }^{10}$ leukosarcoma - a disease characterized by the presence of multiple lymphoid tumors, the cells of which are poured into the blood in such numbers as to constitute a true leukemia. It is also confirmed by Ellermann's ${ }^{11}$ observation that definite lymphoid tumors not infrequently develop in different situations in chronic lymphatic leukemia in both man and fowl.

\section{SUMMARY AND CONCLUSICNS}

1. In view of the fact that one of the principal functions of the lymph nodes is that of a filter, it is reasonably to be assumed that, in a great variety of circumstances, the nodes are subjected to the destructive action of irritants, not only in the forms of solid bodies, such as

10. Sternberg: Beitr. z. path. Anat. u. Allg. Path., 1905, 37, 437.

11. Ellermann: Jour. Am. Med. Assn., 1917, 69, 500. 
bacteria, but as soluble substances of many descriptions. In the case of bacteria, the reaction in the lymph nodes differs with the type of micro-organism and is shown by simple phagocytosis and by regenerative and necrotic lesions, including, in the case of the tubercle bacillus, definite attempts to limit the sphere of activity of the invading parasite by the interposition of a mechanical obstacle in the form of a tubercle. The reaction to soluble poisons is different, as shown, for example, by diffusion of the toxin of the tubercle bacillus, which is followed by hyperplasia of lymphoid cells, sometimes to a limited extent, at other times involving groups of lymph nodes, at still other times including practically all the lymphoid tissues of the body.

Since the lymphoid tissues are so frequently exposed to the action of bacterial toxins and irritants brought to them in solution from absorptive surfaces of vast extent, and since, in these circumstances, they so often display a definite tendency to respond to irritation by hyperplasia of their cellular constituents, I think it not improbable that a like reaction is to be held responsible for the inception of certain types of disease characterized by overgrowth of lymphoid cells, local or general. If the irritant is withdrawn after the lapse of a reasonable interval of activity the cellular reaction in the lymph node ceases and the hyperplastic process is withdrawn. If, however, the irritant continues to act it appears to be inevitable that the cellular response will also continue. Finally, a time is to be expected at which, either as a result of the activity of the same irritant, or, more probably, as the result of the formation of a self-perpetuating toxin in the lymph nodes concerned, the reaction is converted into a process characterized by indefinite hyperplasia.

In the absence of a better explanation, this, I think, is a not illogical method of accounting for the origin of the lymphosarcomas and for similar otherwise inexplicable lesions of the lymphoid system, including not only certain localized lymphoid hyperplasias, but chronic lymphatic leukemia, pseudoleukemia and the like.

2. Chronic lymphatic leukemia and its companion lesion, pseudoleukemia, together with the familiar examples of lymphosarcoma, present many clinical and anatomic changes in common. The histologic alterations are closely akin; in fact, in many instances, they are indistinguishable one from the other. It is simpler and more natural, I think, to correlate these lesions than it is to strain at the impractical and artificial task of attempting to separate them into clinical and anatomic: entities. For this reason I prefer to group them, together with Mikulicz' disease and the symmetrical conjunctival lymphosarcoma herein described, under the general heading of lymphosarcoma, as follows: 
1. Symmetrical superficial lymphosarcoma $\left\{\begin{array}{c}\text { (a) Mikulicz' disease } \\ \text { (b) Bilateral conjunctival } \\ \text { lymphosarcoma }\end{array}\right.$

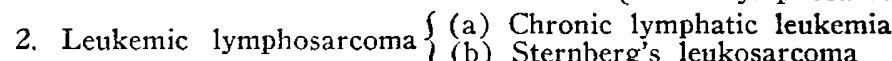

(b) Sternberg's leukosarcoma

3. Regional lymphosarcoma

(a) Cervical, axillary, inguinal

(b) Thoracic $\{$ Thymic

(c) Abdominal $\left\{\begin{array}{l}\text { Peribronchial } \\ \text { Gastro-intestinal }\end{array}\right.$

4. Generalized lymphosarcomatosis

$\left\{\begin{array}{l}\text { Intestinal } \\ \text { Mesenteric, retroperitoneal }\end{array}\right.$

I wish to acknowledge my indebtedness to Dr. Louis Shapiro of the intern staff of Bellevue Hospital for valuable assistance in abstracting a number of clinical facts from the hospital records. 